\title{
ONE-STEP METHODS OF PERIODONTITIS TREATMENT - REVIEW OF THE LITERATURE
}

\author{
Denitsa Zaneva-Hristova, Tsvetelina Borisova-Papancheva
}

\author{
Department of Conservative Dentistry and Oral Pathology, Faculty of Dental Medicine, \\ Medical University of Varna
}

\begin{abstract}
INTRODUCTION: Due to the fact that most of the endodontic problems are of microbial origin, their removal is considered the most important step in root canal therapy. The number of visits to treat infected root canals is a largely discussed topic in endodontics. For that reason, an alternative protocol, including one visit, is proposed.

MATERIALS AND METHODS: The aim of this paper is to investigate the effectiveness of the one-stage treatment of apical periodontitis and to follow the healing process.

RESULTS: After reviewing all articles and studies, we find that the one-visit treatment is considered a good alternative to the endodontic treatment. This is due to the introduction of new and advanced technologies and materials in endodontics, including microscopes, NiTi instruments, newer and more reliable detection devices, isolation and modern obturator systems. Most of the presented studies conclude that there are statistically significant differences based on gender, age, position of the tooth in the jaw relative to the healing process.
\end{abstract}

CONCLUSION: The available evidence based on a systematic review of literature suggests that endodontic treatment of teeth diagnosed with asymptomatic apical periodontitis in one visit may be feasible in certain cases.

Keywords: apical periodontitis, one-visit method, treatment, healing process, postoperative pain, microbiology

Address for correspondence:

Denitsa Zaneva-Hristova

Department of Conservative Dentistry and Oral Pathology

Faculty of Dental Medicine

Medical University of Varna

84 Tzar Osvoboditel Blvd

9000 Varna

e-mail:denicazaneva@gmail.com

Received: December 3, 2017

Accepted: December 29, 2017

\section{INTRODUCTION}

The multiple-visit endodontic treatment is an old and established concept in clinical practice. However, there are still controversies as to if this option for root canal treatment has to be followed with every possible patient and every possible case. Many people believe that the endodontic therapy in one visit is as good an alternative and should be used when applicable. 
One-Step Methods of Periodontitis Treatment - Review of the Literature

Oliet offers certain criteria, the Oliet criteria (1), for an appropriate selection of cases for single-visit endodontic therapy, which include: positive patient acceptance of this endodontic therapy; sufficient time for the proper completion of the procedure; lack of acute symptoms such as pain, swelling and drainage through the root canals; absence of anatomical obstructions such as calcified roots, subtle distorted canals, branched or lateral roots and procedural difficulties such as the formation of thresholds, plugs and perforations.

\section{MATERIALS AND METHODS}

The one-visit root canal treatment concept is described as early as the 1880 s (2-4).

A questionnaire, send by Richard L. Calhoun and R. Randal Landers (5) to endodontists in the United States to investigate their opinion on endodontic therapy in one visit, shows that a greater number of endodontics practice that particular therapy.

Many studies have been made on the basis of a microbiological study, as samples are taken before and immediately after the chemical and mechanical treatment of the root canals.

One of these studies is by T. Kvist (6) in 2004, where 96 teeth with apical periodontitis are studied. The team examines the presence of microorganisms in the root canals after single-visit treatment using a 10 -minute dressing with $5 \%$ iodine-potassium iodide and compares it with a two-visit treatment involving a calcium hydroxide paste. Cultivation of the root canal microorganisms is done before, immediately after the instrumentation, and after the drug insertion. Initial sampling shows the presence of microorganisms in $98 \%$ of the teeth. Postinstrumental sampling reveals a decrease in the cultivated microflora. The antibacterial dressing further reduces the number of teeth with microorganisms. Residual microorganisms in the single-visit treatment are $29 \%$. From a microbiological point of view, the authors conclude that after the treatment of teeth with apical periodontitis performed applying a one-step method, good healing results are expected.

R. Weiger et al. (2000) (7) examine 36 teeth with endodontically induced lesions. They treat the respective teeth with the one-visit treatment meth- od. The criteria for success are the lack of signs and symptoms showing acute phase of periapical periodontitis and radiographically healing of space of the periodontal ligament. The success rate is over $90 \%$. From a microbiological point of view, they find that treatment of root canals in one visit creates favorable environmental conditions for periapical recovery. The team considers that this therapy method is an acceptable alternative to treatment in two visits.

In 1998, Sjögren U (8) and his team examine the role of the infection in the prognosis of endodontic therapy by tracking the teeth 's roots that have been treated and obturated in one visit. The study includes 55 teeth with apical periodontitis. Samples are taken after root canal processing. In 22 of the examined teeth microorganisms are found. The following results are observed 5 years after the initial treatment. Complete periapical cure is observed in $94 \%$ of cases where no bacteria are isolated after root canal treatment. A healing process is observed in $68 \%$ of the teeth in which the microorganisms are isolated. These findings highlight the importance of completely removing the bacteria from the root canals before filling them. They found that this could not be achieved using a one-visit treatment. Removal of the entire infection from the root canal requires an antimicrobial dressing.

A number of studies have been conducted on the basis of postoperative pain as a result of a one-visit method of treating teeth with apical periodontitis:

In 2009, Feroze Ali Kalhoro (9) examines the presence of postoperative pain after treatment of 100 teeth in a one-visit method. They record clinical factors such as patient age, sex, type of tooth, preoperative status of periapical tissues, and postoperative pain and swelling after 1 day, 7 days and 1 month. The significance of the results is obtained by applying a pair of samples t-test and a Pearson X2 test. Three out of 100 cases are characterized with postoperative pain. The remaining patients do not report pain during the study period. After the study, they conclude that root canal treatment in one visit is safe with regard to the postoperative complications.

Ahmed F (10) and his team believe that successful endodontic treatment depends on localization, proper chemiomechanical preparation of the 
Denitsa Zaneva-Hristova, Tsvetelina Borisova-Papancheva

root canal system, shaping, disinfection and threedimensional obturation of the canal system. They report that the disadvantage of one-visit endodontic treatment is associated with postoperative pain and treatment failure. Reducing these disadvantages is possible through the use of new technologies that the team describes. Using the many cases available, they conclude that the outcome of the one-step treatment is favorable when using additional magnification, lighting, laser, and proper irrigation protocol.

Salma Jabeen et al. (11) conduct another study to examine the incidence of postoperative pain after single-phase treatment. Of the 60 patients in the study, 37 have no pain, 12 patients have mild pain, and 11 patients have moderate pain the day after the root canal obturation. On day 7 after the treatment, 50 patients have no pain, 8 patients have mild pain, and 2 patients have moderate pain. No one shows severe pain during the two days of obtaining results. They indicate that the incidence of pain is greater on the first day after obturation and then tends to decrease.

In their study in 1998, Paul D. Eleazer et al. (12) examine 201 teeth that have been treated in one visit. Postoperative complications are observed in 6 teeth of the examined 201 (3\%).

In another study (13) from 2014, Amy WY Wong and her team include low-energy laser photodynamic therapy in the routine endodontic treatment. They investigate the treatment of periodontitis by a one-stage and multiple-visits method. The results obtained after using the laser are inconclusive and further research is needed to show whether the laser should be used in endodontic treatment. They conclude that neither endodontic treatment in one visit nor treatment in multiple visits can guarantee the absence of postoperative pain.

Lara Figini et al. (2008) (14) investigate the effectiveness of single-phase endodontic treatment, measured as teeth extraction due to endodontic postoperative problems. The authors examine the following results: presence of X-ray changes after 1 year, postoperative discomfort, swelling, analgesic use. They find that a complication, such as swelling and intake of painkillers, is observed more frequently in teeth with one-stage endodontic treatment.
JI Edionwe (15) aims to determine the success rate of root canal treatment in one visit. The study includes teeth with irreversible pulpitis, pulp necrosis and apical periodontitis. Patients are observed for 6 months. The teeth included in the study are 40 in 21 women and 24 men from 18 to 56 years old $(34.4 \pm 12.7)$. Of them, $40 \%$ have irreversible pulpitis and $60 \%$ have apical periodontitis. Postoperative pain is reduced to $51.1 \%$ on the first day, $15.5 \%$ after 1 week and $0 \%$ for the remainder of the period. The favorable result of $33.3 \%$ is noted on the first day and rises to $90.5 \%$ at the sixth month. The sixmonth success rate for teeth with irreversible pulpitis is $100 \%$ and $83.3 \%$ for apical periodontitis. The team also takes into account the average time needed to complete endodontic treatment in one visit. To treat a single-crowned tooth, 77 minutes are needed as time increases with the number of roots, reaching 132 minutes for four roots. The team finds that endodontic treatment in one visit improves the prognosis of therapy.

Sumita Bhagwat and Deepil Mehta (16) conduct a study to compare postoperative pain after a onestep method of treatment of non-vital teeth with or without periapical lesions. The results obtained are as follows: in non-vital teeth without periapical lesion $64 \%$ of the cases have pain, $48 \%$ of whom have mild pain and $16 \%$ have moderate pain. In non-vital teeth with periapical changes $32 \%$ of cases have pain, $24 \%$ of whom have mild pain and $8 \%$ have moderate pain. None of the patients report severe pain. Nonvital teeth with periapical lesions show relatively less pain compared to teeth without periapical changes.

In 2009, Feroze Ali Kalhoro (17) aims to determine the incidence of postoperative complications after single-stage endodontic treatment and related factors. For each treated tooth the clinical factors and conditions existing before and after the treatment are recorded. This data include the patient's age, sex, type of tooth, and preoperative status of periapical tissues. They report postoperative pain and swelling after 1 day, 7 days and 1 month after endodontic treatment. The significance of the results is obtained by applying the t-test and the Pearson X2 test. Of the 100 tested teeth, 3 of them have postoperative symptoms. In the remaining 97 cases no pain and edema during the study period are observed. 
The Karen G. Karakov (18) study looks at the effectiveness of using antimicrobial photodynamic therapy in the treatment of chronic periodontitis. The team examines 88 root canals in 84 patients diagnosed based on anamnesis, data from clinical and instrumental examination and X-ray images. They find that the use of antimicrobial photodynamic therapy reduces the incidence of postoperative pain following a one-stage treatment of chronic periodontitis compared to patients treated with a calcium hydroxide preparation. The conclusion of their study is that laser radiation allows to reduce the number of complications by almost 1.5 times, speeding up the process of restoring the outbreaks of bone destruction, allowing for endodontic treatment in one visit.

Syed Gufran Ali's study (19) concentrates on the incidence of pain after root canal treatment, treated using a one-visit method, and evaluates the impact of factors that affect the pain. The study includes 1328 patients who receive endodontic treatment in one visit. Postoperative pain is recorded by each patient using a visual analogue scale at the $12^{\text {th }}$ hour, the $24^{\text {th }}$ hour and the $48^{\text {th }}$ hour after the root canal obturation. After 12 hours, pain is found in $9 \%$ of cases. In the next hours of pain recording the number of teeth with postoperative complications is reduced. After 48 hours, only $4 \%$ of cases feel pain. According to the study, important prognostic factors of pain after root canal obturation are: age, sex and the presence of preoperative pain. Postoperative complications are observed more frequently in adult and female patients. The pulp condition does not affect the intensity and incidence of pain after the root canal is overloaded.

Maria Stella Moreira et al. (2017) (20) research the published systematic reviews comparing endodontic treatment with a one-step method. For this purpose, they use MEDLINE/PubMed electronic databases and Cochrane Registered Controlled Registry by 18 August 2016 and examine the effectiveness of endodontically treated teeth by a one-visit method. Apical periodontitis teeth treated in a single visit show a weak positive trend towards reduced incidence of postoperative complications and higher treatment efficiency.
Another indication used to appreciate the success of one-step endodontic treatment is the healing process traced by radiography.

In 2014, Ipsita Maity (21) assesses the outcome of the one-visit root canal treatment with a diagnosis of apical periodontitis. The team examines ten maxillary frontal teeth showing a periapical lesion on $\mathrm{X}$-ray. The average lesion size varies from 1.3 to 1.9 $\mathrm{cm}$. Postoperative healing processes are observed 3 months, 6 months and 1 year after treatment using subjective feedback and radiography. It was found that by the end of 6-12 months of treatment, 8 out of 10 cases show signs of a complete healing process.

Field JW and his team (22) conduct a clinical $\mathrm{X}$-ray retrospective assessment of the success rate of root canal treatment in one visit. They observe 768 cases examined in one visit, of which 223 are followed over a period of 6 months to 4 years from the day of treatment. The overall success rate is $89.2 \%$. The team does not observe statistically significant differences based on gender, age, and position of the tooth in the jaw. Statistically, the outcome for frontal teeth is better than for the teeth in the distal region.

As early as 1986, Richard B. Pekruhn (23) investigates the failure after endodontic therapy in one visit and found a minimal failure rate of $5.2 \%$ or 48 of the 925 teeth tested.

Joseph J (24) examines the success rate after endodontic therapy in one visit by examining 210 teeth. 102 of them are tracked in time for the healing process. The evaluation of the condition of the treated teeth consists of patients' subjective data, clinical examination and diagnostic radiographs. A case is considered to be successful if there are no clinical symptoms and the following radiographic criteria are met: the preceding lesion is completely cured or reduced in size. From the total number of 102 traced teeth, 91 or $89 \%$ are considered to be treated successfully.

Boggia (25) examines teeth with a diagnosis of apical periodontitis by treating them with a one-visit method via endometasone paste. A complete periapical healing is observed when the $\mathrm{X}$-ray results are tracked.

In their study, Martin Trope et al. (26) evaluate the radiographic healing of teeth with apical periodontitis treated in one or in two visits with or without calcium hydroxide as a temporary intracanal 
Denitsa Zaneva-Hristova, Tsvetelina Borisova-Papancheva

medication. Through the Periapical Index (PAI), they compare the differences in periapical status from the start of the treatment to the $52^{\text {nd }}$ week. They manage to find that the periapical status of the endodontically treated teeth improves significantly after the stated period. The group of teeth treated with a multiphase calcium hydroxide application method shows the greatest healing process using PAI. The weakest healing processes are observed in the group treated with a multiple-visit method without the use of intracanal medication. The teeth treated in a one-step method occupy an intermediate position in view of the healing process reported by the periapical index.

In 2011, Mansoor Khan and his team (27) publish reports of two cases of acute apical abscess. Their goal is to prove the effectiveness of endodontic treatment in one visit. Case tracking shows symptom relief to a remarkable extent in the evening after treatment. X-rays establish a significant healing process in the periapical tissues after 3 months in both patients.

In the study of Ali M. Rashid (28), 965 cases are investigated, of which 322 are traced back to the rehabilitation process. Statistical analysis is performed using the Chi-square test and variants of failure rates based on gender, type of tooth, position and jaw are considered. A t-test is used to assess age data. Results are obtained in which the success rate is $92.8 \%$. They do not detect statistically significant differences based on gender and jaw. The data show that in younger patients the failure rate is higher compared to older patients, and that frontal teeth achievement is greater than the teeth in the distal region.

At the Peking University School of Stomatology in China, Ming-Ming Zhang and his team (29) scan ESC 93 single-crowned teeth one year after one-visit endodontic treatment. They use a Wilcoxon test to evaluate one-year and two-year volumetric changes after treatment. Thrity-two of the teeth examined have a recovery process at the first scan, one year after treatment. In 61 of the tested teeth, a second scan is needed, two years after the treatment. The total number of lesions significantly decreases in the second year. The volume of the changes decreases in 38 teeth (63\%), remains unchanged in 20 (33\%) and increases in 2 teeth (3\%). They conclude that the volume of posttreatment changes found 1 year after treatment is significantly reduced after the second year in $63 \%$ of the teeth.

Peters LB et al. (2002) (30) aim to evaluate the treatment of periapical lesions in single sessions in the presence or absence of microorganisms. They examine 21 teeth filled with gutta-percha and AH-26 sealer after microbiological sampling. The teeth are traced over a period of up to 4.5 years for periapical radiolucency. After the studies, the authors establish full X-ray treatment in $81 \%$ of the cases in the group with one visit. The likelihood of success increases continuously over time.

In the Paredes-Vieyra J study (31), 155 teeth with apical periodontitis are treated using a one-visit method of therapy. Diagnosis is confirmed by radiographic data and by a negative response to hot and cold tests. Nine teeth out of all 155 are lost in tracking time. Two years later a healing process is observed in $96.57 \%$ (141 of 146 teeth) in the group of teeth treated in one visit.

In 2008, Anders Molander and his team (32) study 53 teeth for endodontic treatment in one visit. At the end of their study, 32 teeth (65\%) are classified as cured. The team concludes that good therapeutic results can be obtained through single-visit antimicrobial therapy.

\section{DISCUSSION}

The presented studies show that the one-step method of treating apical periodontitis is possible and to a large extend proven to be successful. To be such, however, certain clinical criteria should be met such as lack of pain, swelling and drainage through the root canals; lack of anatomical obstacles such as calcified or subtle distorted canals and procedural difficulties such as the formation of cracks, stoppers and perforations. Another important criterion is to spend enough time to properly complete the manipulation within one visit. It has been shown that 77 minutes are required for the treatment of one-root tooth, and the time increases proportionately to the number of root canals, reaching 132 minutes for four.

Complete periapical healing in one visit is illustrated in a number of the articles presented, evaluating the success rate using radiographic criteria such as absence or size reduction of preceding periapical lesion as well as lack of clinical symptoms. The likelihood of success increases continuously over time as

Scripta Scientifica Medicinae Dentalis, 2017;3(2):29-35 
there is a certain regularity in the postoperative complications. These are observed more often in adult and female patients. The condition of the pulp does not affect the intensity and incidence of pain after the root canal is overloaded. The outcome in frontal teeth is better than in the teeth in the distal region. Teeth with periapical changes show relatively less pain than teeth without periapical lesions.

According to most of the studies, the root canal treatment in one visit is safe with regard to the postoperative complication.

\section{CONCLUSION}

The available evidence, based on a systematic review of literature, suggests that endodontic treatment of teeth diagnosed with asymptomatic apical periodontitis in one visit may be feasible in certain cases. Apical periodontitis teeth treated in a single visit show a weak positive trend towards reduced incidence of postoperative complications and higher treatment efficiency.

\section{REFERENCES}

1. Oliet S. Single-visit endodontics: a clinical study. J Endod. 1983;9(4):147-52. doi: 10.1016/ S0099-2399(83)80036-3.

2. Dodge JS. Immediate root filling. Dent Cosmos. 1887;29(4):234-5.

3. Kells CE. Immediate root filling. Dent Cosmos. 1887;29(6):366-7.

4. Turner WJ. New York Odontological Society. Dent Cosmos. 1901;43:1405-12.

5. Calhoun RL, Landers RR. One-appointment endodontic therapy: a nationwide survey of endodontists. J Endod. 1982;8(1):35-40. doi: 10.1016/ S0099-2399(82)80315-4.

6. Kvist T, Molander A, Dahlén G, Reit C. Microbiological evaluation of one- and two-visit endodontic treatment of teeth with apical periodontitis: a randomized, clinical trial. J Endod. 2004;30(8):572-6.

7. Weiger R, Rosendahl R, Löst C. Influence of calcium hydroxide intracanal dressings on the prognosis of teeth with endodontically induced periapical lesions. Int Endod J. 2000;33(3):219-26.

8. 8. Sjögren U, Figdor D, Persson S, Sundqvist G. Influence of infection at the time of root filling on the outcome of endodontic treatment of teeth with apical periodontitis. Int Endod J. 1997;30(5):297-306.
9. Kalhoro FA, Mirza AJ. A study of flare-ups following single-visit root canal treatment in endodontic patients. J Coll Physicians Surg Pak. 2009;19(7):4102. doi: 07.2009/JCPSP.410412.

10. Ahmed F, Thosar N, Baliga MS, Rathi N. Singlevisit endodontic therapy: A review. Austin J Dent. 2016; 3(2):1035.

11. Khurshiduzzaman SJ. A study of post obturation pain following single visit root canal treatment. Chattagram Maa-O-Shishu Hospital Medical College Journal. 2013;12(3):16-9. doi:10.3329/cmoshmcj.v12i3.16707.

12. Eleazer PD, Eleazer KR. Flare-up rate in pulpally necrotic molars in one-visit versus two-visit endodontic treatment. J Endod. 1998;24(9):614-6. doi: 10.1016/S0099-2399(98)80122-2.

13. Wong AW, Zhang $\mathrm{C}$, $\mathrm{Chu} \mathrm{CH}$. A systematic review of nonsurgical single-visit versus multiple-visit endodontic treatment. Clin Cosmet Investig Dent. 2014;6:45-56 . doi: 10.2147/CCIDE.S61487.

14. Figini L, Lodi G, Gorni F, Gagliani M. Single versus multiple visits for endodontic treatment of permanent teeth: a Cochrane systematic review. J Endod. 2008;34(9):1041-7. doi: 10.1016/j.joen.2008.06.009.

15. Edionwe JI, Shaba OP, Umesi DC. Single visit root canal treatment: a prospective study. Niger J Clin Pract. 2014;17(3):276-81. doi: 10.4103/1119-3077.130210.

16. Bhagwat $S$, Mehta $D$. Incidence of post-operative pain following single visit endodontics in vital and non-vital teeth: An in vivo study. Contemp Clin Dent. 2013;4(3):295-302. doi: 10.4103/0976-237X.118352.

17. Kalhoro FA, Mirza AJ. A study of flare-ups following single-visit root canal treatment in endodontic patients. J Coll Physicians Surg Pak. 2009 Jul;19(7):410-2. doi: 07.2009/JCPSP.410412.

18. Karakov KG, Gandylyan KS, Khachaturyan EE, Vlasova TN, Oganyan AV, Eremenko AV. Comparative characteristics of the methods of treatment of chronic periodontitis using antibacterial photodynamic therapy (per one visit) and Calasept preparation. J Natl Med Assoc. 2018 Feb;110(1):73-77. doi: 10.1016/j.jnma.2017.01.013.

19. Ali SG, Mulay S, Palekar A, Sejpal D, Joshi A, Gufran $\mathrm{H}$. Prevalence of and factors affecting post-obturation pain following single visit root canal treatment in Indian population: A prospective, random- 
Denitsa Zaneva-Hristova, Tsvetelina Borisova-Papancheva

ized clinical trial. Contemp Clin Dent. 2012; 3(4): 459-63. doi: 10.4103/0976-237X.107440.

20. Moreira MS, Anuar ASN, Tedesco TK, Dos Santos M, Morimoto S. Endodontic treatment in single and multiple visits: An overview of systematic reviews. J Endod. 2017;43(6):864-70. doi: 10.1016/j. joen.2017.01.021.

21. Maity I, Meena N, Kumari RA. Single visit nonsurgical endodontic therapy for periapical cysts: A clinical study. Contemp Clin Dent. 2014;5(2):195202. doi: 10.4103/0976-237X.132321.

22. Field JW, Gutmann JL, Solomon ES, Rakusin H. A clinical radiographic retrospective assessment of the success rate of single-visit root canal treatment. Int Endod J. 2004;37(1):70-82.

23. Pekruhn RB. The incidence of failure following single-visit endodontic therapy. J Endod. 1986;12(2):68-72. doi: 10.1016/ S0099-2399(86)80131-5.

24. Jurcak JJ, Bellizzi R, Loushine RJ. Successful single-visit endodontics during Operation Desert Shield. J Endod. 1993;19(8):412-3. doi: 10.1016/ S0099-2399(06)81507-4.

25. Boggia R. A single-visit treatment of septic root canals using periapically extruded endomethasone. Br Dent J. 1983;155(9):300-5. doi: 10.1038/ sj.bdj.4805218.

26. Trope M, Delano EO, Orstavik D. Endodontic treatment of teeth with apical periodontitis: Single vs. multivisit treatment. J Endod. 1999;25(5):34550. doi: 10.1016/S0099-2399(06)81169-6.

27. Khan M, Khan RMA, Javed MQ, Ahmed A, Nabeel $M$. Treatment of acute apical abscess by single visit endodontics - 2 case reports. Pak Oral Dent J. 2011;31(1):199-202.

28. Rashid AM. Retrospective Assessment of the success rate of single-visit root canal treatment: A clinical and radiographical Analysis. Al - Rafidain Dent J. 2008;8(1):104-9.

29. Zhang MM, Liang YH, Gao XJ, Jiang L, van der Sluis L, Wu MK. Management of apical periodontitis: healing of post-treatment periapical lesions present 1 year after endodontic treatment. J Endod. 2015;41(7):1020-5. doi: 10.1016/j.joen.2015.02.019.

30. Peters LB, Wesselink PR. Periapical healing of endodontically treated teeth in one and two visits obturated in the presence or absence of detectable microorganisms. Int Endod J. 2002;35(8):660-7.
31. Paredes-Vieyra J, Enriquez FJ. Success rate of single- versus two-visit root canal treatment of teeth with apical periodontitis: a randomized controlled trial. J Endod. 2012;38(9):1164-9. doi: 10.1016/j. joen.2012.05.021.

32. Molander A, Warfvinge J, Reit C, Kvist T. Clinical and Radiographic Evaluation of one- and two-visit endodontic treatment of asymptomatic necrotic teeth with apical periodontitis: A randomized clinical trial. J Endod. 2007;33(10):1145-8. doi:10.1016/j. joen.2007.07.005. 